\title{
Expectation and satiation accounts of ambiguous figure-ground perception
}

\author{
MARTIN S. LINDAUER \\ State University of New York, College at Brockport, Brockport, New York
}

\begin{abstract}
An expectancy account of figure-ground shifts, promoted as an alternative to satiation theory, was tested. One-hundred and three subjects in six conditions (Experiment 1) were either informed or uninformed about 12 to 13 ambiguous figures, and had either unlimited time or $30 \mathrm{sec}$ in which to respond. In two of the conditions, head and eye movements were restricted, and in one condition, incidental learning was used. In keeping with an expectancy view, uninformed subjects saw fewer meaningful shapes than informed subjects did, and restricted eye/head movements played no role. However, in accord with a satiation view, most figures did shift for many subjects. Unexpected for both accounts, inspection time was not a factor. In Experiment 2, 59 uninformed subjects saw two ambiguous figures whose alternative shapes were physically emphasized in order to increase both attention and satiation. Contrary to satiation expectations, but supporting an expectancy position, subjects saw one shape as often as two. The applicability of inherent stimulus factors to figure-ground perception was discussed.
\end{abstract}

In figure-ground perception, two (or more) meaningful shapes within a composite stimulus emerge sequentially, as in the ambiguous Rubin face-vase stimulus. A Gestalt account of these shifts refers to sensory satiation (Kohler \& Wallach, 1944); that is, receptors for each of the alternating components of the figure-ground stimulus differentially fatigue. The satiation position, however, has received little empirical support (Coren \& Girgus, 1978, p. 188; but see Rock, 1984, p. 121). A cognitive account of some of the shifts in figure-ground perception has been offered by Rock (1984, pp. 122-123). Changes are initiated, at least in part, by instructions that typically inform subjects about the ambiguity of the stimulus. Change is therefore expected, looked for, and found. Girgus, Rock, and Egatz (1977) demonstrated that uninstructed subjects reported few figure-ground changes.

The generality of Rock's (1984) position was tested in Experiment 1 with a relatively large set of ambiguous figure-ground stimuli (12-13). Girgus et al. (1977) relied on only 2-3 ambiguous figures, of which only 1 was figure-ground. (The others were reversible figures that shifted in spatial orientation rather than in meaningfulness.) Furthermore, Girgus et al. (1977) measured the number of figure-ground changes over a fixed time period. In the studies reported here, the identified shapes were recorded, indicating whether changes in the percept took place.

The role of time was also explored, by exposing the stimuli for either a relatively short or a relatively long

A briefer version of this paper was read at the annual meeting of the Eastern Psychological Association, Arlington, VA, April 1987. The author wishes to thank Donna Merlo for conducting Conditions 4 and 5 of Experiment 1. Requests for reprints should be addressed to Martin S. Lindauer, Psychology Department, State University of New York, College at Brockport, Brockport, NY 14420. period. According to the satiation theory, the longer the inspection, the greater the neural fatigue, and hence the more rapid appearance of alternate shapes (from "rested" cells). Increased time, however, also allows cognitive factors to play a role: boredom occurs, curiosity emerges, and perceivers have more time to search for additional information. Subjects may therefore "stumble" across the alternate shapes in the stimulus. Thus, for both satiation and expectancy theories, time of inspection should be positively related to the number of figures seen.

Some subjects were informed about the nature of the stimuli (the typical procedure), whereas others were uninformed to varying degrees. Following an expectancy account and contrary to satiation expectations, fewer ambiguous figure-ground shapes would be perceived by uninformed subjects than by informed subjects.

\section{EXPERIMENT 1}

\section{Method}

Booklets containing 13 relatively well-known ambiguous figure-ground stimuli, one on each page, were shown to subjects in six conditions. The stimuli included an old-young woman (from Boring), a rabbit-pirate, a hawk-dove, and assorted faces and bodies, both human and animal (see Figure 1). Reversible figures (e.g., the Necker cube) were excluded since their alternate shapes could not be identified easily by name. The maximum number of shapes that could be seen was 29 (several stimuli contained more than two figures).

In Condition 1, 14 subjects were fully informed about figure-ground perception. The ambiguous Rubin vase-face was explained and used as a sample training stimulus. Furthermore, the identities of the alternate shapes were given in the context of a question printed on each page of the booklet (e.g., "Can you identify the mouth of the old woman?'). (The subjects indicated what they saw by drawing a line from the question about the shape's identification to the shape itself, which they then circled.) Another clue was found in the written and oral instructions, which stated that there was more than one meaningful shape on each page of the booklet. In addition, the subjects were enrolled in a percep- 


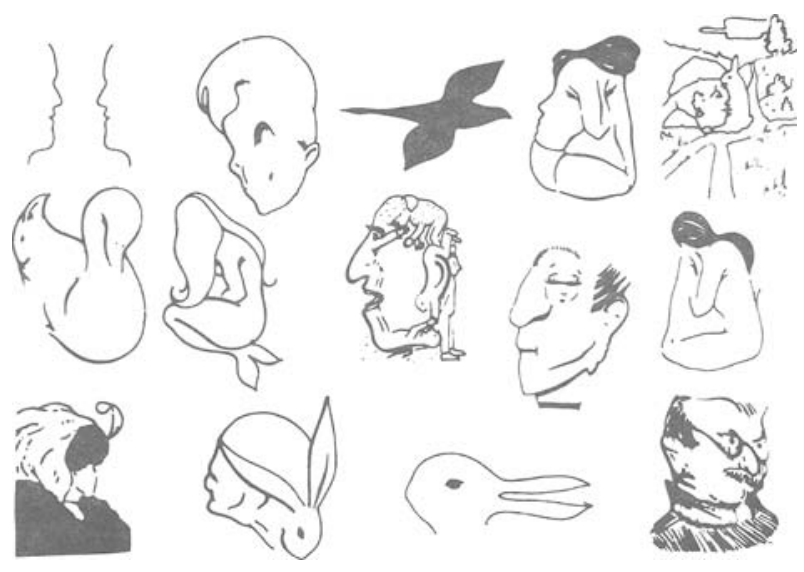

Figure 1. The sample stimulus (the vase-face) and the 13 ambiguous stimuli.

tion course, in which the concept of figure-ground perception was discussed just before the study began. The subjects were tested in a single group session. (In this and the other conditions, females predominated, accounting for $65 \%-75 \%$ of the subjects in each condition.)

In uninformed Conditions 2 and 3 ( $n=19$ and 20, respectively), subjects had less background in psychology, perception, and figure-ground shifts than those in Condition 1. Subjects in Condition 2 were recruited from introductory psychology classes, during the first weeks of the term, before the topic of perception had been discussed. Subjects in Condition 3 were mainly upperclassmen taking an advanced psychology class. Although those who had taken the perception course $(n=5)$ were excluded, many of the students were psychology majors and/or had taken several psychology classes. Thus, they probably had some familiarity with figure-ground perception, more than subjects in Condition 2 did, but less than those in Condition 1. Subjects in both Conditions 2 and 3 had no training on figure-ground perception, no information about the ambiguity of the stimuli, and no indication that more than one shape was present. Their task was to identify what was seen, if anything. Otherwise, the instructions were the same as those given in the informed Condition 1. Subjects in Condition 2 were tested in small groups of 4-6 each, whereas those in Condition 3 were tested in a single group session.

Subjects in Condition 2 could take as much time as they needed to respond; those in Condition 3 were given $30 \mathrm{sec}$. The latter was a longer exposure. In a pilot study of 23 subjects (who received the same instructions), the mean identification time for each stimulus was 21-24 sec (depending on whether outlyers were excluded or not).

Subjects in Conditions 4 and 5 ( $n=21$ and 19, respectively), unlike those in Conditions 1-3 (and 6), were individually tested. Participants were alternatively assigned to Condition 4 or Condition 5 , depending on their order of appearance at the lab. Like all others, they were volunteers (for credit) from the introductory psychology courses. The figure-ground stimuli were shown on slides that projected a $15 \times 11$ in. image $(37.5 \times 27.5 \mathrm{~cm})$ located $66 \mathrm{in} .(165 \mathrm{~cm})$ in front of the subjects (visual angle $=13.02^{\circ}$ ). The stimuli were shown in one of 16 random orders (selected from a table), and each was seen for $30 \mathrm{sec}$ (as in Condition 3). Only 12 of the 13 stimulus items could be shown, since subjects would have had to invert one stimulus (the bald man) in order to see both forms. (In order to make the data of Conditions 4 and 5 comparable to the other conditions, a score of 1 was added to each subject's total response. This score approximates the typical response to the bald man stimulus, since very few subjects [1-2] in the other conditions had seen either none or both of the shapes of this stimulus.)

Each stimulus in Conditions 4 and 5 was preceded by a fixation point, and the subjects' head and eye movements were restricted (with a chinrest and head restraint). These conditions, meant to increase the num- ber of receptor cells stimulated (compared with the free viewing of the other conditions), were expected to maximize any satiation effect.

Instructions for Conditions 4 and 5 reduced the possible expectation that there would be more than one shape in each stimulus. Thus, in Condition 4 , the instructions used general terms to refer to the multiple shapes that might be found in the set of stimuli, rather than explicitly stating (as the earlier instructions did) that each slide could contain one or more shapes: "The slides contain shapes of various things ... [that] are fairly well hidden and some will be hard to recognize. Thus, it's possible you won't see anything identifiable..."' The instructions in Condition 5 went even further by adding that "The slides may contain just random lines and filled areas, or they may contain one or more meaningful and recognizable shapes.... Thus, it's possible that the slides won't contain anything identifiable, or they might have one or two or even more recognizable shapes..."

The debriefing in Conditions 4 and 5 also differed from that in the other conditions. Information about the subjects' nonresponses was obtained by showing them some of the stimuli whose alternate shapes had not been reported. (The number of stimuli shown depended on the time available in the allotted 45-min session.) The subjects were then asked, to the best of their recollection, if the omitted shapes had not been seen or not been reported.

In Condition 6, expectancies were minimized even further by presenting the 13 ambiguous figure-ground stimuli under incidental conditions. Fifteen introductory psychology students were tested as a group during their first week of introductory psychology, before they had read or heard anything about perception or figure-ground shifts (or had participated in any studies). They were given the same booklet used in the informed Condition 1; however, nothing was said about the stimuli or their multiple shapes (although subjects could read about them in the booklets). The subjects were told that the booklets, previously used in another study, would be used in a later study. In order to determine the booklets' usefulness, they had to be checked to make sure the pages were "clean." Booklets with used pages were to be noted and put aside.

The scanning of the booklets took no more than $5 \mathrm{~min}$ (the time varied somewhat for different subjects). (The total time for Condition 3, in which subjects saw each stimulus for $30 \mathrm{sec}$, was $6.5 \mathrm{~min}$.) When finished, and with the booklets closed, the subjects were then unexpectedly asked to list the meaningful objects, shapes, or forms they might have noticed, if any, while examining the booklets. Following this incidental task, the subjects were told about the ambiguity of the stimuli. They then were told to reexamine the booklets, and to find and identify as many shapes as they could.

\section{Results}

In Condition 1, the only condition in which subjects were fully informed about figure-ground shifts, significantly more shapes were seen than in Conditions 2, 3, 4 , and $5[M=26.21$ vs. $17.09,18.95,19.19$, and 19.63, respectively; $F(4,84)=14.82, p<.01]$. Condition 1 significantly differed from each of the other four uninformed conditions $[t(28-38)=6.15-8.38, p<.01$; different $d f \mathrm{~s}$ are due to varying $n \mathrm{~s}]$. The four uninformed groups did not differ from one another $[t(33-38) \leq 1.70$, $p>$.05]. In addition, subjects in Condition 1 identified more of the two (or more) components that made up the 13 sets of hidden shapes than did subjects in the other conditions $[M=11.07$ vs. $4.06-5.86 ; F(4,84)=16.39$, $p<.01 ; t(28-38)=6.60-8.38, p<.01]$. The uninformed Conditions 2-5 again did not differ from one another $[t(33-38) \leq 1.92, p>.05]$.

The fewest shapes were identified under the incidental circumstances of Condition $6(M=4.94$, median = 5.00 ), significantly fewer than any of the other five unin- 
formed Conditions 2-5 [t(27-34) $\geq 8.33, p<.01]$. However, when subjects were instructed about the task, the mean number identified rose to 25.60 , which is about the same as in the informed Condition $1(M=26.21)$.

The exact number of shapes seen and reported is ambiguous, however. A particular label (e.g., "face') could describe several stimuli, be a synonym for a response already made, or represent a guess for stimuli that were not fully identified. (The few descriptions not obviously descriptive of any of the possible 13 figures-e.g., "a house" -were excluded.) The number of reported figures was probably reduced by memory loss, or inflated because some subjects read the instructions and did the study. Thus, both underestimations and overestimations existed, and they might have cancelled each other out. However, the incidental circumstances must have minimized any expectations about figure-ground shifts, and hence contributed to the reduced number of alternative shapes seen.

Aside from the instructions, there are other possible sources of expectation. One may have come from the first response to the stimuli, in which either one or both shapes were seen. However, the initial view did not arouse any expectations about the multiple nature of subsequent stimuli. To demonstrate this, the data of Conditions 4 and 5 were examined, since these were the only conditions in which each of the stimuli was presented first (in one of the random orders used). Subjects were divided into two groups: those who reported either one or two (or more) shapes in the first stimulus. The first response to the set of stimuli did not affect the total number of subsequent shapes seen $[M=19.50$ and 18.95 , respectively, $t(37)=1.11, p>.05]$. Thus, even if more than one shape was initially seen, subjects did not expect multiple shapes in subsequent stimuli.

Nor was a general familiarity with figure-ground shifts, from courses in psychology, a source of expectations. The most informed subjects (Condition 3) were advanced students with several nonperceptual courses in psychology. Despite some acquaintance with figure-ground perception, at least from their introductory course, they identified the same number of ambiguous shapes as did introductory students with little knowledge of psychology (Conditions 2, 4, 5, and the informed Condition 6).

The reports of what was seen were not suppressed by response inhibition. This was determined from the retrospective accounts of subjects in Conditions 4 and 5 . (The group testing of the other conditions prevented individual debriefing.) After examples of the unreported shapes were pointed out, the subjects were asked if the alternative shapes had been seen earlier but not reported. The subjects were nearly unanimous in asserting that what they said reflected what they saw, and most expressed surprise when told that there were several shapes present. (In the pilot study, only one person indicated that more was seen than was said.) Admittedly, these retrospective accounts must be cautiously interpreted, since they are biased by memory and experimenter demands.
In summary: Shifts in figure-ground perception were reduced when information about the possibility of change was neutral. The time available (either unlimited or $30 \mathrm{sec}$ ) was irrelevant, as were the testing circumstances (group or individual), viewing restrictions (free or limited head and eye movements), and the subjects' backgrounds in perception.

\section{EXPERIMENT 2}

According to a cognitive perspective (Rock, 1984), stability in figure-ground perception could also be increased by holding the perceiver's attention, that is, by not allowing it to waver or be directed elsewhere because of boredom or curiosity. However, according to satiation theory (Kohler \& Wallach, 1944), the opposite should occur: holding attention should increase reversals because more cells would be stimulated longer and hence fatigued sooner.

In Experiment 2, components of one of the alternate shapes in an ambiguous figure-ground stimulus were physically emphasized, and at the same time, aspects of the alternative shape were weakened. With one shape more distinctive, both attention and satiation should be simultaneously increased (Lindauer, 1969). However, the effects should be divergent: depending on whether satiation or attention was increased, figure-ground shifts should either occur or not occur, respectively.

\section{Method}

Two ambiguous stimuli from the previous experiment were used: the pirate-rabbit and the face-mermaid. The rabbit and face were the single responses that predominated (in 14 and 10 instances, respectively) in the uninformed Condition 2 (with no time limit) of Experiment 1, which is comparable to this experiment.

Physical emphasis was achieved by darkening the mouth of the face or the tail of the mermaid of the face-mermaid stimulus, and the hat of the pirate or the ears of the rabbit of the pirate-rabbit stimulus. Weakening included lightening an area and leaving out details. Despite the sharpening or blurring of details, however, both versions of the ambiguous figure could still be seen (as the results indicate).

Either one of the two figure-ground stimuli was alternately shown first, and the two physically altered versions of each figure were presented successively. For example, some subjects first saw one version of the pirate-rabbit figure, and then the alternate version of the same figure; this pair was then followed by the two versions of the mermaid-face figure. The order of the stimuli shown and the version shown first alternated for each individually tested subject.

Booklets with the stimuli were shown individually to 59 undergraduate volunteers (for credit) from several introductory psychology classes. The task was presented as "a break" in the middle of an unrelated study. The instructions (uninformed) and procedures (self-paced) were identical to the earlier Condition 2, whose data serves as the expected baseline response.

\section{Results}

In general, when only one shape was reported, it was most often (in $80 \%$ of the cases) the physically emphasized component of the ambiguous figure $\left[\chi^{2}(1)=\right.$ 4.48-14.73, $p<.05 ; n=11-22$ ]. This pattern varied, however, depending on the stimulus shown and its order. When the mermaid-face was shown first (before the 
pirate-rabbit), the response was a single shape in $66 \%$ of both physically altered versions of this stimulus $\left[\chi^{2}(1)\right.$ $=4.17-6.53, p<.05 ; n=24-30]$. (The $n s$ here and below varied because some subjects reported seeing no figures.) When the mermaid-face stimulus was shown second (after the pirate-rabbit stimulus), either one or both shapes was reported equally often $\left[\chi^{2}(1)<1 ; n=28\right]$. Response equivalence was also found in both versions of the rabbit-pirate stimulus, whether the stimulus was shown first or second (after the mermaid-face): seeing either both shapes or a single one was equally likely [in $52 \%$ and $48 \%$ of the cases, $\chi^{2}(1)<1, n=22-25$ ].

A more detailed analysis revealed that the favored shape depended on the particular aspect of the ambiguous figure that was physically altered. Thus, when the pirate shape of the pirate-rabbit ambiguous figure was physically dominant, the pirate was seen by $92 \%$ of the 23 subjects who saw one figure (only 2 subjects saw the rabbit); 24 subjects saw both shapes, and the remaining 10 subjects saw neither. These results differed significantly from those reported in Condition 2 of Experiment 1, in which the (untouched) rabbit shape rather than the pirate was more frequently reported $\left[\chi^{2}(3)=31.02, p<.05 ; n=78\right]$. When the rabbit shape was physically prominent, it was the most frequently reported shape, and there was no difference from the frequencies in Condition $2\left[\chi^{2}(3)=\right.$ $3.51, p>.05 ; n=78]$. This was not unexpected since the unaltered rabbit was the dominant single shape reported then.

As for the mermaid-face figure, when the face was made physically prominent, it was favored by $64 \%$ of the subjects who gave a single response (23 of 36 subjects); about one-third of the subjects (36\%) saw the mermaid, and the other 23 subjects saw both shapes. This response pattern differed from Condition 2 of Experiment $1\left[\chi^{2}(3)\right.$ $=24.37, p<.01 ; n=78$ ]. Even though the face was also the more frequent response earlier, it was more dominant in Experiment 2. The response pattern for the physically emphasized mermaid, however, did not differ from its earlier profile in Condition $2\left[\chi^{2}(3)=5.91\right.$, $p>.05 ; n=78]$.

\section{GENERAL DISCUSSION}

The findings of Experiments 1 and 2 largely confirm Rock's (1984) expectancy predictions. The results of Experiment 1 parallel those of Girgus et al. (1977), but with a different measure (the identification of the shapes seen), and with a larger number of ambiguous figure-ground stimuli (12-13). The shapes did not automatically shift from their initial meanings unless change was expected (Condition 1 and the informed posttest of Condition 6). When subjects did not expect the stimuli to be ambiguous (Conditions 2-5 and the incidental task of Condition 6), there were significantly fewer shifts. The results of Experiment 2 were also congruent with an expectancy position. When attention was held by giving physical prominence to one of the two key areas of the ambiguous stimuli, shifts occurred less often than when the ambiguous shapes were physically equivalent (Condition 2 of Experiment 1).

For satiation theory, the uninformed (and incidental) circumstances of Experiment 1 should have been irrelevant, since neural process, and hence figure-ground shifts, are automatic. Also, a satiation account would have expected the physical enhancement in Experiment 2 to have increased, rather than reduced, the amount of change.

The data, however, do not completely favor an expectancy position. Although the number of figures seen under the informed and the uninformed conditions differed, the two (or more) aspects of most figures were seen by more than half of the subjects. For example, in Conditions 4 and 5 , the alternate shapes in all but two of the stimuli were seen by two-thirds to three-fourths of the subjects. The component shapes of the old-young woman and the dove-hawk were the most difficult to disambiguate, but even in these cases, $54 \%-58 \%$ of the subjects saw both figures. (These rates differ somewhat across the different conditions.)

These differences between the stimuli were probably the result of their inherent or structural characteristics (Coren \& Girgus, 1978), such as dark-light contrasts and the amount of filled and unfilled areas (Lindauer \& Lindauer, 1970). These structural distinctions, along with expectancy, may help account for the ease or difficulty with which the alternate shapes of different stimuli were seen (Lindauer \& Baust, 1974).

Problematic for both the expectancy and the satiation positions was the equivalence in the number of shifts under either self-paced or 30sec exposure (Conditions 2 and 3-5, respectively). A satiation account would have predicted more figure-ground changes with the controlled (and longer, on the average) exposure time. As the initially stimulated cells fatigued, more alternate shapes should have been seen. However, the expectancy position would have made the same prediction. During the long (and forced) inspection time, subjects were likely to become bored and to look for more stimulation. Consequently, the likelihood of seeing an alternative shape should have been increased. The issue needs to be addressed with greater control over, and a broader range of, exposure times.

\section{REFERENCES}

Coren, S., \& Girgus, J. S. (1978). Seeing is deceiving: The psychology of visual illusions. Hillsdale, NJ: Erlbaum.

Girgus, J. J., Rock, I., \& Egatz, R. (1977). The effect of knowledge of reversibility on the reversibility of ambiguous figures. Perception \& Psychophysics, 22, 550-556.

Kohler, W., \& Wallach, H. (1944). Figure after-effect: An investigation of visual processes. Proceedings of the American Philosophical Society, 88, 269-357.

LiNDAUER, M. S. (1969). Set and different degrees of ambiguity of the "wife and mother-in-law" figures. Perceptual \& Motor Skills, 29, 911-913.

Lindauer, M. S., \& Baust, R. F. (1974). Comparisons between 25 reversible and ambiguous figures on measures of latency, duration, and fluctuation. Behavior Research Methods \& Instrumentation, 6, 1-9.

Lindauer, M. S., \& Lindauer, J. F. (1970). Brightness differences and the perception of figure-ground. Journal of Experimental Psychology, 84, 291-295.

Rock, I. (1984). Perception. New York: Scientific American.

(Manuscript received September 19, 1988.) 